Instability, Chaos and Predictability in Celestial Mechanics and Stellar Dynamics
Copyright 1992 by Nova Science Publishers, Inc. All rights of reproduction in any form reserved. ISBN 1-56072-054-9

\title{
ON INSTABILITY LEADING TO CHAOS IN DYNAMICAL SYSTEMS
}

\author{
L.M. Saha \\ Department of Mathematics, Zakir Husain College, \\ Ajmeri Gate, New Delhi - 110006, India
}

\section{ABSTRACT}

Instability of orbits in dynamical systems leading to chaos has been reviewed briefly. Stability criteria for some unimodal mapping which provide various periodic regimes during the period doubling bifurcations has been discussed in deta 11 . Stability conditions are also reviewed for standard map lor Chirikov-Taylor map), and results obtained for range of values of the non-linear parameter appearing in the map have been studied. Strange attractor has al so been discussed.

\section{INTRODUCTION}

Studies on nonlinear dynamics and emergence of chaos are of growing interest at the present time. Chaotic phenomena have brought new mathematical ideas and analytical technique. The subject is fascinating because of its interplay of sience, mathematics and technology. In past tho decades scientists of various disciplines, (e.g. Ref.[1]-[11],[13]-[19],[54]), have come to the common conclusion that "a simple system may give rise to a complex behaviour and a complex system may give rise to a simple behaviour". Almost all nonl inear systems exhibit chaotic motion and so, chaotic phenomena are a wide class of natural events found in the physical world. Chaos happens more frequently than order. Chaos is a science of computer age. Modern computational technique and elegant mathematics made chaos to emerge as one of the most exciting and intriguing areas of cience. Therefore, the investigations on chaos has brought a great revolution to modern scientists. Chaos has provided tools to explain evolution of dynamical systems to a vast range of real phenomena. 
Hadamard (1898), [33], wa $\mathrm{f}$ ir st to observe sensitive dependence of chotic trajectories on initial conditions which was also realized, later on, by Duhem (1906), [34], a nd Po incare (1908), [36],[31]. Poincare observed that la fully deterministic dynamic s does not necessarily imply an expl ic it prediction on the evolution of a dynamical system". Poincare's observation again came to light, after a long gap and with great excitement, by variou s researc hers, ([1], [2], [6]-[9], $[12],[13],[19]-[33],[37]-[53],[56]$, (Ref.[38],[40],[41] together known as a KAM theory)l and a theory of dymamical chaos wa born.

Stability of a nonlinear system is very compl icated than in the 1 inear case. Here one has to go through its local as well as global a spects. The chaotic motion in a dynamical system is a realt of dynamic instability of orbits in the system. Stability of motion of a nonl inear system which contains some parameters, say $\lambda$, may change in the vicinity of a $f$ ixed point. The $f$ ix ed point becomes unstable when $\lambda$ attains a critical value and a $2-c y c l e$ is born. This $2-c y c l e$ becomes unstable when $\lambda$ further $c$ hanges and attains an a nother critical value and a $4-c y c l e$ is born and so on. Thus we see that whenever a stable motion becomes unstable a bifurcation starts and a period doubling phenomenon occurs, ([1],[2],[6], $[11],[20],[22],[24]-[28],[37],[45],[48],[51],[52])$. The various critical values of $\lambda$ obey a general rule

$$
\lim _{n \rightarrow \infty} \frac{\lambda_{n}-\lambda_{n-1}}{\lambda_{n+1}-n}+\delta=4.6692016
$$

where $\lambda_{n}$ stand $s$ for critical value of $\lambda$ at the nth bifurcation. $\delta^{n}$ is known as Feigenbaum's constant. From the above procedure one may accurately determine that critical value for $\lambda$ after which the motion turns to be chaotic.

In the present work a brief review has been presented on instability leading to $c$ haos. As an illustration, the popula tion model, al so known as logistic mapping, has been used as a model for the deacrete dynamical system.

\section{DYNAMICAL SYSTEMS, CHAOS AND UNPREDICTABIL ITY}

\subsection{Dynamical system:}

A dynamical system is that who se evolution from some initial state, (prescribed), can be described by some rule(s) in the form of mathematical equations. The evolution of such a system is best described by the so-called pha se space. The dynam ical systems mostly we encou nter are nonlinear in nature whose evolution is controlled by certain parameter which we have already stated. 
Until now, there is no generally accepted definition of chaos. However, a s it appears through recent 1 iteratures, (e.g . Ref.[1],[2],[6]-[9],[13],[16],[19]-[32] etc.), chaos can be well under stood from the following ideas:

i) Chaos is an effect of instability of orbits in a dynamical system.

ii) The phenomenon related to the occurance of randomness and unpredictability in a completely deterministic system is called chaos.

iii) Irregular behaviour in both conservative and dissipative systems is termed as chaos.

iv) Chaotic behaviour simply looks markedly more irregular than regular behaviour.

v) Chaotic trajectories show sensitive dependence on initial conditions i.e., chaotic trajectories show an average exponential divergence of initially near by trajectories.

vi) Chaos describes a situation where typical solutions br orbits) of a differential equation (or typical evolution of some other model determining deterministic evolution) do not converge to a stationary or periodic function bf time) but continue to exhibit a seemingly unpredictable behaviour.

vii) Chaos can be thought as a new regime of nonlinear oscillations, as overlap of resonances, as accumulations of many instabilities, etc.

viii) Chaos implies that the knowledge of initial data is inafficient for long time prediction.

\subsection{Unpredictabil ity}

The instability, non-deterministic behaviour and chaotic motion are consequences of the results of non-accurate predictability of the evolution of a dynamical system. Accurate prediction of the evolution of a dynamical system is extremely difficult as it depends on the facts, [65], that how accurately (a) the principles of dynamics describing such evolution be formulate i.e. the laws governing such evolution, (b) the mathematical model be established ?i.e., the differential equations establ ished how accurately representing the system? (c) the approximation of the solution be made ? (d) the initial conditions and other numerical parameters be used?

The above conditions are very important because a minute error may cause a perfectly deterministic periodic motion to a chaotic one and vice-versa, Szebehely $([61]-[63])$. 


\subsection{Sensitive Dependence on Initial Conditions}

Accuracy in initial conditions is a very important factor because the chaotic trajectories show sensitive dependence on the initial conditions, [66]. This means that for a small change $\delta x(0)$ in the initial condition $x(0): x(0) \rightarrow x(0)$ $+\delta x(0)$, the point $x(t)$ at time $t$ may change as $x(t) \rightarrow x(t)$ $+\delta x^{-}(t)$, such that $\delta x(t) \sim \delta x(0) e^{\Lambda t}$, where $\Lambda$ is call ed the Lyapünov character istic exponent (L.C.E.). Lyapunov exponents are tools to determine whether or not the system is chaotic. The exponential convergence or divergence of initially nearby trajectories provide a conclusive way to distinguish between torus and chaos by estimating Lyapunov exponents $\Lambda_{i}$ : $\Lambda<0$ means stability whereas $\Lambda>0$ stands for unstable behaviour and if $\Lambda_{\max }>0$ the system is defined to be chaotic, [52], and in this case a deterministic motion turns to be a chaotic one (Fig. 1 ).

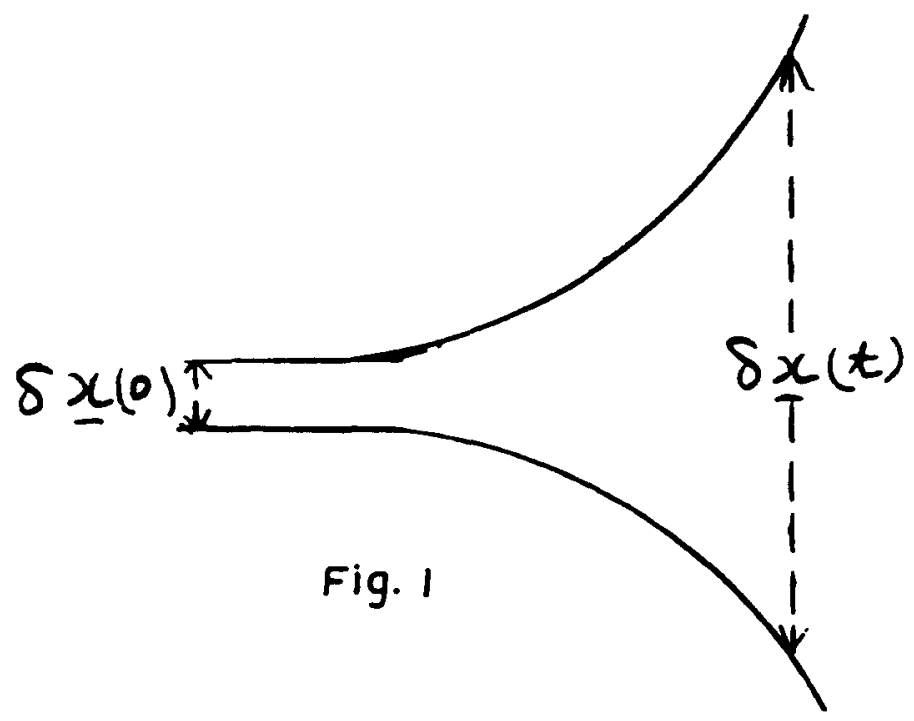

\section{BIFURCATIONS: STABIL ITY OF FIXED POINTS}

The study of bifurcations has developed into a major area of mathematical and applied research. Jacobi ([56]) has u sed the term "branching off " for bifurcation which is due to poincaré, [57]. The term bifurcation mostly now applied to situations involving (i) the study of the change in the mumber 
of $f$ ixed points as the control parameters $\lambda$ are varying and (ii) the dynamic studies concerned with the change in the topology, (the phase portraits), as $\lambda$ are changing. By changing $\lambda$ a bifurcation may occur at any time and the phase portrait may change to a topologically non-equivalent portrait.

Let us consider a descrete dynamical system who se evolution is represented by the deterministic map

$$
x_{n+1}=F\left(x_{n}\right)
$$

The equilibrium value $x^{*}$, for which $x^{*}=F\left(x^{*}\right)$, is said to be the $f$ ixed point of $F$. Then, $x^{*}$ is said to be stable if the sequence of iterates $x_{1}, x_{2}, \ldots, x_{n}, \ldots$ of $F$ converges to $x^{*}$,

$$
\text { i.e. } \quad \lim _{\mathrm{k} \rightarrow \infty} x_{k}=x^{*} \text {, }
$$

for all initial values $x(0)$ of $x$, or alternatively, if

$$
\left|F^{\prime}\left(\lambda, x^{*}\right)\right|=\left|\frac{x_{n+1}-x^{*}}{x_{n}-x^{*}}\right|=\left|\frac{d F}{d x^{*}}\right|<1,
$$

and $x^{*}$ is unstable if $\left|\frac{d F}{d x^{*}}\right|>$.

Figures 2 (a) and 2 (b) respectively representing stable and unstable fixed points.

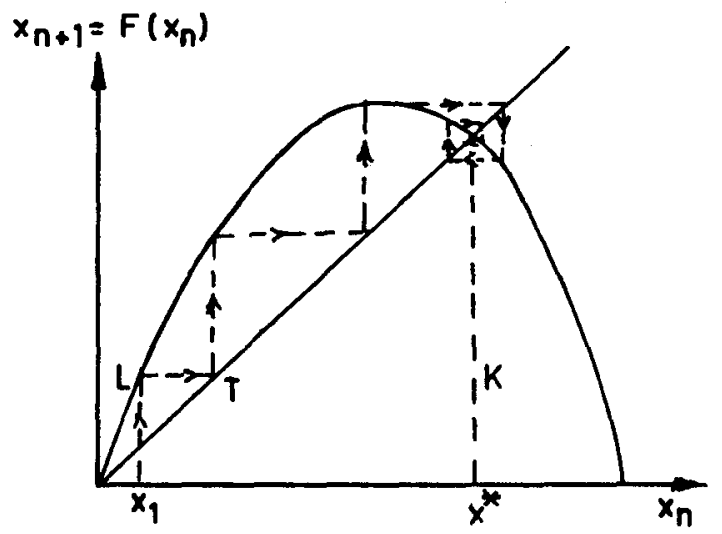

Figure 2 (a): Stable fixed point (Attractor) 


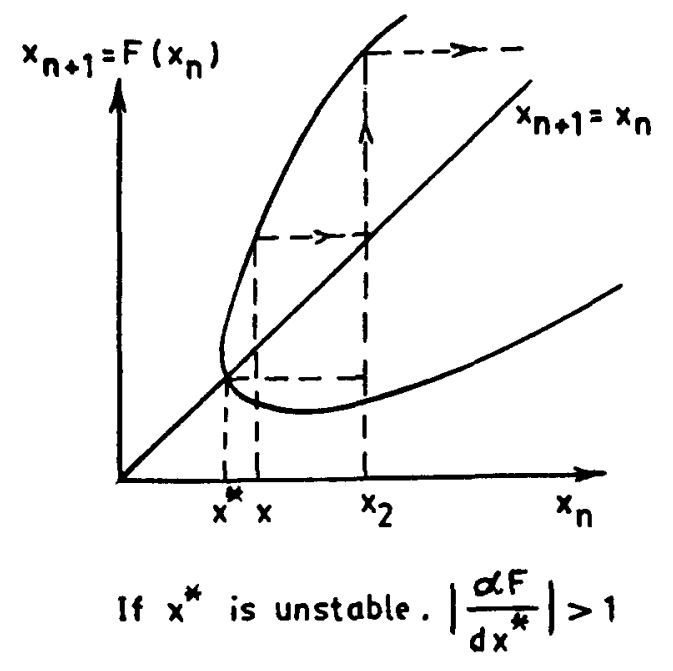

Figure 2 (b): Unstable fixed point

A stable $f$ ixed point is termed as an attractor because the points in its neighbourhood approach to it when iterated.

As stated earlier, when the controlling parameters $\lambda$ of $F$ vary and attain some critical value, say $\lambda_{c}$, stability of $x^{*}$ is disturbed and bifurcation starts and we observe a period doubling scenario, [ 6],[11],[12],[24]-[30],[37],[51], [52]), of which a periodic pattern of period $p$ or a p-cycle is defined by

$$
x_{i+p}=x_{i}, \quad x_{i+k} \neq x_{i} \text {, for all } k<p,
$$

and $i$ greater than certain $\mathrm{N}$.

Thas, $p=1$ corresponds to the fixed point $x^{*}$. An attractor $x^{\star}$ is not onl $y$ the isolated point, there might be $p-$ point in $p-c y c l e, x_{1}^{\star}, x_{2}^{\star}, \ldots, x_{p}^{*}$ such that

$$
\begin{aligned}
& x_{i+1}^{*}=F\left(x_{i}^{*}\right), \quad i=1,2, \ldots, p-1 \\
& x_{1}^{*}=F\left(x_{p}^{*}\right)
\end{aligned}
$$

The set $\left\{x_{1}^{*}, x_{2}^{\star}, \ldots, x_{p}^{*}\right\}$ is called a p-point 1 imit cycle.The p-cycle is stable if 


$$
\left|\prod_{i=1}^{p} F^{\prime}\left(\lambda, x_{i}^{*}\right)\right|<1 \text {, }
$$

where the chain rule of differentiation has been used. That is, the p-point limit cycle is stable if each $x_{i}^{*}$ in $\left\{x_{1}^{*}, x_{2}^{*}, \ldots\right.$

If the set $\left\{x_{i}^{*}\right\}, i=1,2, \ldots, p$ is a global attractor then for almost every initial point $x_{0}$, the sequence $x_{n}=F(n)$ $\left(x_{0}\right)$ approaches the sequence $x_{1}^{*}, x_{2}^{*}, \ldots, x_{n}^{*}, x_{1}^{*}, x_{2}^{*}, \ldots, x_{n}^{*}, \ldots$

\section{INSTABIL ITY LEADING TO CHAOS}

The sequence of critical values of $\lambda$ where bifurcations occur obey the general rule (1.1). It has been observed that the 1 imiting value $\delta$ in $(1.1)$ approaches by third or fourth bifurcation. So by changing $\lambda$ one may accurately determine that critical value of $\lambda$ after which the motion turns to be chaotic.

To illustrate chaotic behaviour, Feigenbaum and many others ([24]-[28],[45], etc.) have u sed population model, [67], which is also known as logistic mapping and is written as

$$
f(x)=\lambda x(1-x),
$$

$x=0$ and $x=\frac{\lambda-1}{\lambda}$ are $f$ ixed points of $f$. The period two points of $f$, including the $f$ ixed points of $f$, are given by the roots of the equation

$$
f^{2}(x)-x=0
$$

or

$$
[f(x)-x]\left[\lambda^{2} x^{2}-\lambda(\lambda+1) x+(\lambda+1)\right]=0 .
$$

Thus, the period two points of $f$ (not fixed points of $f$ ) are given by

$$
\frac{\lambda+1+\sqrt{(\lambda-3)(\lambda+1)}}{2 \lambda} \text { and } \frac{(\lambda+1)-\sqrt{(\lambda-3)(\lambda+1)}}{2 \lambda}
$$

Now if (i) $\lambda<3$, there are no such real points,

(ii) $\lambda_{\text {of }} f=3$, there is exactly one point, the $f$ ixed point

and (iii) $\lambda>3$, there are two different points.

Thus a point two or $2-c y c l e$ is created as $\lambda$ increased through 3. Also, note that the $f$ ixed point is attracting for $\lambda<3$ and 
repelling for $\lambda>3$ and the attracting nature is passed on to the period two cycle which exists only for $\lambda>3$ and is attracting. We can illustrate this by plotting $f$ and $f^{2}$ against $x$ for various values of $\lambda$, (above, below and equal to 3 ) Figure 3 (a) and 3 (b) are such plottings.
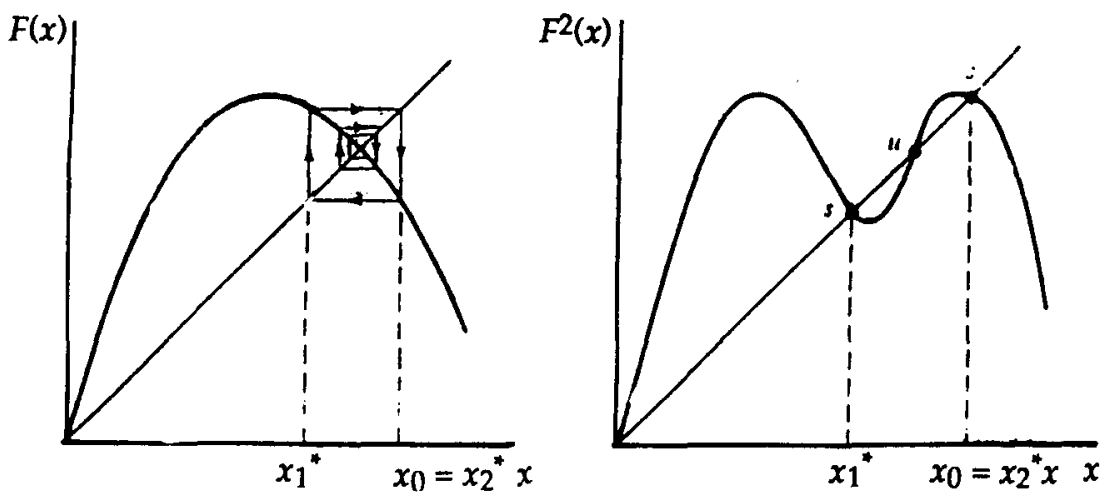

Figure 3 (a) and $3(b)$

As $\lambda$ increases further, the $2-c y c l e$ becomes repelling and an attractive 4-cycle is born; then a $8-c y c l e$ and so on and we observe the period doubling scenario. For example

$$
\begin{aligned}
& \lambda=2.9 \text { gives an attractive } 1-c y c l e \\
& \lambda=3.1 \text { gives an attractive } 2-c y c l e \\
& \lambda=3.5 \text { gives an attractive } 4-c y c l e \\
& \lambda=3.56 \text { gives an attractive } 8-c y c l e \\
& \lambda=3.566 \text { gives an attractive } 16-c y c l e
\end{aligned}
$$

i.e.. there is a set $\left\{\lambda_{c}\right\}$ of critical values of $\lambda$ sach that if $\lambda_{c_{n}}<\lambda<\lambda_{c_{n+1}}$ there is a stable period $2^{n}-c y c l e$ and $\lim _{n \rightarrow \infty} \lambda_{c_{n}}=\lambda_{\infty}$, (finite). In the control phase space the $a b-$ ove period doubling bifurcations look like Fig. 4. The \pm sign for branches indicate that each bifurcation point $\bar{x}_{\text {(n) }}$ can be associated with its branch by a \pm subscript, (e.g. (n) $=+,+,-,+,-,-$ ). 


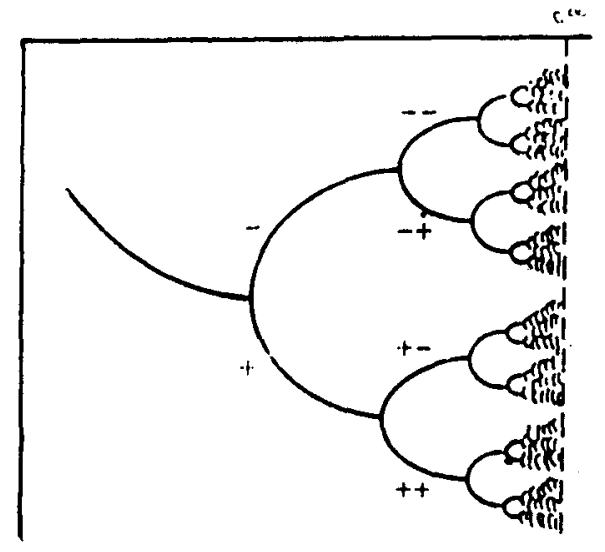

Figure 4:

It has been observed that beyond the critical values of for which motion show period doubling, chaotic motion exist in a band of parameter values. When these bands are of finite width, periodic windows be developed for the parameter within which the motion may aga in undergo period doubling bifurcation and again leading to chaotic motion, [6],[11],[12],[51], [52]). Thus in the above example as $\lambda$ increases to 4, at various values of $\lambda$, an attractive $q-c y c l e$ is born followed immediately by period doubling sequence of attractive $2 q, 4 q$, $8 \mathrm{~g}, \ldots$ cycles.

When $\lambda>4$, then there is a cantor set $J$ in $[0,1]$ arch that (i) $x \in J$ iff $f(x) \in J$, (ii) $f^{n}(x) \rightarrow-\infty$ if $x \notin J$ and (iii) $f$ has a chaotic action on $J$.

Therefore, we can conclude that the period doubling phenomenon is the most celebrated scenario for chaotic motion.

In case of the logistic mapping [45], written as $x_{n}=$ $1-\lambda x_{n-1}^{2}$, the period doubling scenario appears at critical values of $\lambda$ given by

$$
\begin{aligned}
& \mathrm{p}=1,0<\lambda<\lambda_{\mathrm{c}_{1}}=0.75 \\
& \mathrm{p}=2, \lambda_{\mathrm{c}_{1}}<\lambda<\lambda_{c_{2}}=1.25 \\
& \mathrm{p}=4, \lambda_{c_{2}}<\lambda<\lambda_{c_{3}}=1.3680989 \\
& \mathrm{p}=8, \lambda_{c_{3}}<\lambda<\lambda_{c_{3}}=1.3940461
\end{aligned}
$$


which quickly converges to an aperiodic orbit at $n \rightarrow \infty$, the value $\lambda_{\infty}=1.401155 \ldots$ In the range $\left(\lambda_{\infty} 2\right)$ there exists an infinite number of periodic windows immersed in the background of aperiodic regime. Figure 5 represent bifurcation diagram for the mapping $x_{n+1}=\lambda-x_{n}^{2}$, Ref. [37], [68].

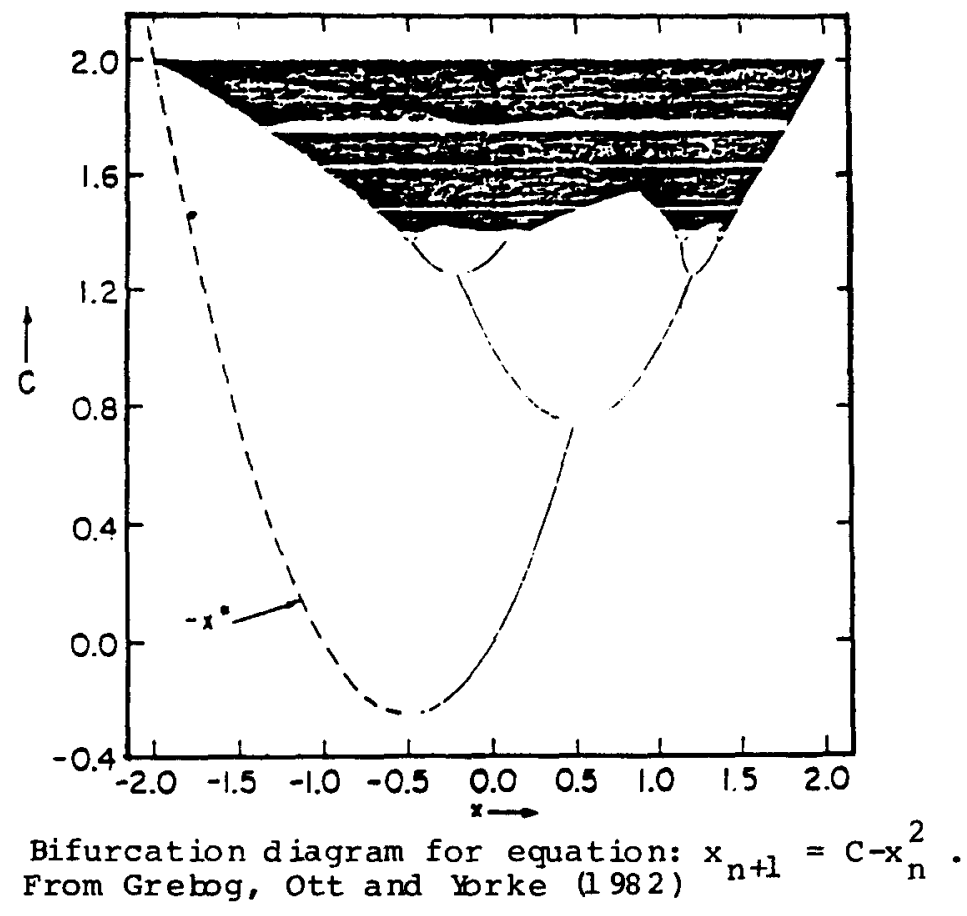

The problem of turbulence is a long standing phenomenon of physics and as it appears in recent literature, ([22],[25] $[27],[50],[5]],[52],[55])$, the chaotic phenomena are rel evant to the onset mechanism of turbulence. When the Reynold's mumber $R$ is small enough, the fluid flow is laminar and statioary corresponding to a suitable fixed point in its phase space. However, when $R$ is increa sed to a certain critical value $R_{C^{\prime}}$ the $f$ ixed point is no more stable, for of attracting nature) and a stable limit cycle closed around the fixed point be established. When $R$ again be increa sed to a nother critical value $R_{c_{2}}$, the 1 imit cycle loses its stability and a stable 2-Torus, (an attracting clo sed tube), around the unstable 1 imit cycle be established and so on. Landau and bpf, ([58]-[60]), identified the final state of this infinite process with an infinite number of incommensurable frequencies as fully turbulent. This is known as Landau-bpf route to turbulence. Later on, $([50],[51])$, it has been showed that 
three consecutive bifuractions are enough to have erratic motion by interweaving trajectories attracted to a low dimensional manifold in the same phase space called strange attractor. The motion on strange attractors be identified with turbulence. Thus, in the scheme we have,

Fixed Point $\rightarrow$ Limit Cycle $\rightarrow$ 2-Torus $\rightarrow$ Strange Attractor Chaos

This concludes that the qua siperiodic motion on a. 2-Torus may have loose stability and may give birth to chaos direct$1 y$.

\section{STRANGE ATTRACTOR: ([ 23$],[2],[7],[24]-[28],[56])$}

As stated earlier a stable $f$ ixed point is an attractor. An attracting 2-cycle is the stable two-period cycle and, in general, an attracting $p-c y c l e$ means stable p-period cycle.

We say that the dexcrete dynamical system posses chaos if it has some strange attractor. Strange attractors are mathematical objects but computers have given them 1 ife and draw pictures of them. A strange attractor is first an attractor which consists of an infinity of points in the plane of $\mathrm{m}-\mathrm{d} i$ mensional space. These points correspond to the state of a chaotic motion. he call it "strange" as it has the structure of a fractal set. The inter section of this object with a straight line results in a "Cantor set". It has an infinitely nested structure which can be seen in each repeated minor magnification.

A strange attractor is defined for a map $f$ as an infinite point set $\Omega$ such that (i) $f(\Omega)=\Omega$, (ii) $f$ has an or bit which is dense in $\Omega$ and (iii)' $\Omega$ has a neighbourhood $N$ consisting of points who or bits tends a symptotically to i.e., $1 \mathrm{im} f(n)(N) \subset \Omega$. Orbit in or near such 1 imit set be$n \rightarrow \infty$

have in an essentially chaotic manner.

Hénon's attractor [7] and Lorenz's attractors [23] are examples of strange attractors.

\section{CONCLUDING REMARKS}

What has been revealed through this brief revien indicates that to study nonlinear phenomena in more realistic way one must use the chaotic theory. Finally, let us recall the statement of Ian Stewart, [54], that chaos is (i) exciting as it provides tools for simplifying complicated phenomena, (ii) worrying becau se it introduces new doubts about the traditional model building procedure, (iii) fascinating for its interplay of mathematics, science and technology and (iv) beautiful, above all. 


\section{ACKNOWL EDGEMENTS}

The author wishes to present his sincere thanks to Prof. K.B. Bhatnagar who extended his help and encouragement in preparation of this paper.

\section{REFERENCES}

[1] Ruelle, D. (1989), Chaotic evolution and strange attractors, Cambridge University Press, Cambridge.

[2] Dno, Y. and osikawa, M. (1980), Prog. Theor. Phys.,64, $54-67$.

[3] Goldberger, A.L., Rigney, D.R. and West, B.J. (1990), Sci. Amer., February, 35-4l.

[4] McRobie, A. and Thompson, M. (1990), New Scientist, 9 June, 41 .

[5] Lesurf, J. (1990), New Scientist, 30 June, 63 .

[6] Guckenheimer, J. and Holmes, P. (1983), Nonl inear oscillations, Dynamical systems and Bifurcations of Vector Fields, Springer-Verlag, New York.

[7] Hémon, M. (1 976), Comm. Math. Phys., 50, 69-79.

[8] Chirikov, B.V. (1979), Phys. Reports, 52, $263-379$.

[9] Helleman, R.H.G. (1980), Fundamental Problems of Statistical Mechanics, Ed. Cohen, E.G.D., North-tblla nd Publ.. Am sterdam, 5, 165-233.

[10] May, R.M. (1974), Ścience, 186, 645-647.

[11] May, R.M. (1976), Nature, 261, 459 .

[12] Li, T. and Yorke, J.A. (1 975), Amer. Math. Monthly, 82, $985-992$.

[13] Moser, J. (1986), SIAM Reviews, 28, 459.

[14] Tsonis, A.A. and El sner, J.B. (1988), Nature, 333,545547 .

[15] Tsonis, A.A. (1 989), Chaos and unpredictability of weather.

[16] Moon, F.C. (1987), Chaotic vibrations, J.Wiley and Sons, New York.

[17] Palmer, T. (1 989), New Scientist, 11 Nov. 56-59.

[18] Scott, S. (1989), New Scientist, 2 December, 53-59.

[19] Moser, J. (1973), Stable and Ra ndom Motions in Dynam ical systems, Princeton University Press, Princeton, N.J.

[20] Cook, P.A. (1986), Nonl inear Dynamical Systems, Prentice Hell Internationa1, New Del hi.

[21] Hénon, M. and Heiles, C. (1964), Astrn. J. 69, 7.3-79.

[22] Hao, B.L. (1 984$),$ Chao s. World Scientif ic Pu $\overline{\mathrm{Bl}}$., Singa pore.

[23] Lorenz, E.N. (1 963), J.Atmos. Sci., 20, 130-141.

[24] Feigenbaum, M.J. (1978), J.Stat. Phys., 19, 25-52 (1979) J.Stat. Phys., 21, 669-706. 
[25] Feigenbaum, M.J. (1980), Comm. Maths. Phys., 77, 6586.

[26] Feigenbaum, M.J. (1982), Lecture Notes in Physics, Ed. Carrido, L., 179, Proceedings, Stiges, 131-148.

[ 27 ] Feigenbaum, M.J. (1979), Phys., Lett. 74A, 375.

[28] Feigenbaum, M.J., Kada noff, L.P. a nd shenker, S.J., (1982), Physica, 5D, 370 .

[29] Feigenbaum, M.J., Jensen, M.H. and Procacci, A.I. (1 980$)$, Phys. Rev. Lett. 57, 1503 .

[30] Ruelle, D. (1990), Proc. Roy. Soc. Iond., A427, 241 248.

[31] Carrido, L, and Simo, C. (1982), Lectures Notes in Physics, md. Carrido, L.,179, Proceedings, Stiges, 127 .

[32] Benettin, G., Froschle, C.and Scheidecker, J.P. (1 979), Phys. Rev., Al 9, 2454.

[33] Padamard, J. (1898), J. Math. Pure Appl.. 4, 23-73.

[34] Duhen, P. (1906), La theorie physique. Soñ object et sa structure, Paris: Chevalier et Riviere.

[35] Poincare, H. (1908), Sciences et Methode.

[36] Metropolis, N., Stein, M. and Stein, P.R. (1973), J. $\mathrm{J}$. Combinatorial Theo.., 15, 25-44.

[37] Kolmogorov, A.N. (1954), Les Alamas scientif ic Laboratory Translation, LA-TR-71-76 by Dahly, H. of Akad.

Nauk. SSSR Doklady 93, 763, (1 953), Doklady Akad. Nauk. $98,527$ (1 954$)$.

[38] Arnold, V.I. (1961), Izv. Akad. Nauk. SssR, Ser.Mat., 25,21 .

[39] Arnold, V.I. (1963), U spekhi Mat. Nauk., 18, 6, 91 .

[40] Moser, J. (1962), Nach. Akad. Wiss. Gottingen Math. Phys., K1, 21.

[41] Moser, J. (1968), Lectures on Hamiltonian Systems. $M \in$. AM $\leq 81$.

[42] Arnold, V.I. . and Avez, V. (1968), Ergodic Problems of Classical Mechanics, Benjamin, New York.

[43] Fermi, E., Pasta, J. and Ulam, S. (1 965), Los Alamas Report LA-1 940: The collected papers by E.Fermi, University of Chicago Press, 2, 978 .

[44] Collet, P. and Eckmann, J.P. (1 975), Iterated Maps on the interval as dynamical systems, Birkhau ser.

[45] Hao, B. -L. (1 983), Prog. Phys., 3, 329.

[46] Yi-sui-gun (1 985), Celes. Mech., 37, 171-181.

[47] Sharkovski, A.N. (1964), Ukx. Math. Zurn., 16, 61-71.

[48] Ostlund, S., Rand, D., Sethna, J.P. and Siggía, E.D. (1983), Physica, 89, 303 .

[49] Arnold, V.I. (1 978), Mathematical Methods of Classical Mechanics, Springer, New York.

[50] Ruelle, D. and Takens, F. (1 971), Comm. Math. Phys., 20, $167-192$.

[51] Newhouse, S., Ruelle, D. and Takens, F. (1 978), Comm. Math. Phys., 64, 35-40. 
[52] Takens, F. (1981), Lecture Notes in Mathematics, Springer, 898, 712-716.

[53] Vivaldi, F. (1989), New Scientist, 28 Oct. 46-49.

[54] Stewart, I. (1 989 ), New Scientist, 4 Nov.., 42-46.

[55] Kaplan, J.L. and Yorke, J.A. (I 979), Ann. N.Y. Acad. Sci., Vol. 316.

[56] Jacobi, C. (1834), Prog. Ann., 32, 229.

[57] Poincare, H. (1885), Acta Math., 7, 259-380.

[58] Landau, L.D. (1 944 ), Dokl. Akad. Nauk. SSSR, 44,339342 .

[59] Landau, L.D. and Lif shitz, E.M. (1 959), Fluid Mechanics, Oxford, Pergamon.

[60] Hpf, E. (1948), Comm. Pure Appl. Math., 1, $303-322$.

[61] Szebehely, V.(1 986), Particle Accelerators, 19, $43-47$.

[62] Szebehely, V. (1 986), New Non-deterministic celestial Mechanics, D.Reidel Co.Dordrecht, Holland.

[63] Szebehely, V. (1988), Celest Mech., 43, 139-145.

[64] Eichhorn, H. (1987), Celest, Mech., 43, 418 .

[65] Marchal, C. (1987), Proc. 96th IAU Coll. Ed.Valtonen, M.J., Turku, Finland, 91-97.

[66] Richard son, L.F. (1948), Psychometrika, 13, 147-174.

[67] Grebogi, C., Ott, E. and Yorke, J.A. (1 98र), Phys.Rev. Lett., 50, 935-938. 